\title{
XII. Beitrag zur krystallographischen Kenntniss des Bournonit nebst einem Anhange: Wärmeleitung des Antimonit und Bournonit.
}

Von

\author{
F. B. Peck in München.
}

(Mit Tafel II u. 1 Textfigur.)

Bekanntlich hat Miers $\left.{ }^{1}\right)$ die Isodimorphie der normalen Sulfarsenite und Sulfantimonite von der Formel $A s S_{3} A g_{3}$ und $S b S_{3} A g_{3}$ nachgewiesen, nachdem vorausgehend Luedeck e ${ }^{2}$ ) die monokline Natur der Feuerblende festgestellt hatte. Die beiden zu dieser Gruppe gehörigen monoklinen Substanzen haben die folgenden Elemente:

$$
\begin{aligned}
& \text { Xanthokon } a: b: c=1,9187: 1: 1,0152 ; \beta=88^{04} 7^{\prime} \\
& \text { Feuerblende } a: b: c=1,9465: 1: 1,0973 ; \beta=90 \quad 0
\end{aligned}
$$

Da nun Bournonit ebenfalls die Zusammensetzung eines normalen sulfantimonigsauren Salzes besitzt, wie die Feuerblende, nur dass in demselben das $A g$ theilweise durch $P b$, theilweise durch $C u$ vertreten ist, so liegt es nahe, zu vermuthen, dass zwischen der Krystallform beider gewisse Beziehungen existiren.

Betrachtet man die obigen Elemente, so sieht man, dass die Krystallformen der betreffenden Mineralien dem rhombischen System ausserordentlich nahe: stehen (bei der Feuerblende ist die Axenschiefe so gering, dass sie nicht bestimmt werden kann), und das Axenverhältniss sehr nahe $=$ 2:1:1 ist, während in dem Axenverhältnisse des Bournonit, wie er gewöhnlich gestellt wird,

$$
a: b: c=0,9379: 1: 0,8968 \text { ist. }
$$

Nimmt man fürl die Feuerblende ein halb so grosses $a$, so erhalten wir:

$$
a: b: c=0,9732: 1: 1,0973 \text {. }
$$

1) Diese Zeitschr, 22, 433 .

2) Ebenda 6, 372 . 
Nehmen wir fermer beim Bournonit das bisherige $\{001\}(c)$ als $\{100\}$ und das bishcrige $\{010\}(b)$ als $\{001\}$, so wird das Axenverhalıniss:

$$
a: b: c=0,9561: 1: 1,0662 \text {, }
$$

wodurch die Aehnlichkeit der beiden Mineralien in ihren Axenverhältnissen klar hervortritt.

Die oben hervorgehobene chemische und krystallographische Verwandtschaft des Bournonit mit den monoklinen Gliedern dieser Gruppe legte die Möglichkeit nahe, dass derselbe ebenfalls monoklin sei.

Um in dieser Frage Klarheit zu schaffen, wurde eine erneute sorgfältige Untersuchung des Bournonit unternommen. Es dienten hierzu $\mathbf{4 6}$ isolirte Krystalle, welche zum grössten Theile gut messbar waren. 32 wurden vollständig durcbgemessen, nämlich :

$$
\begin{aligned}
9 & \text { von Pribram, } \\
3 & \text { - Silberwiese bei Ems, } \\
2 & \text { - Horbausen, } \\
1 & \text { - Offenbanya, } \\
10 & \text { - Nagyag, } \\
7 & \text { - Kapnik. }
\end{aligned}
$$

Diese Krystalle befinden sich theils in der mineralogischen Sammlung in Múnchen, theils stammten sie aus den Sammlungen in Wien, Prag und Přibram, welche zu benutzen mir ebenfalls gestattet wurde.

Wählen wir die vorhin erwähnte Stellung, so müsste im Falle des monoklinen Systems der Winkel $c: b$ eine Abweichung von $90^{\circ}$ zeigen und die bisher als $\{011\}$ betrachtete Form $n$ in zwei ungleichwerthige Flächenpaare zerfallen. Da die vorherrschende Fläche $c$ stets uneben ausgebildet ist, kann die Frage nur entschieden werden durch den Nachweis constanter,

\begin{tabular}{|c|c|c|c|c|c|c|}
\hline & & & $n:$ & & $n^{\prime}:$ & \\
\hline Krystall & Nr. 1 & Pribl'am & $48^{\circ}$ & $3^{\prime}$ & $48^{0}$ & $7^{\prime}$ \\
\hline- & -3 & $3-$ & 48 & 10 & 48 & 5 \\
\hline - & $-\quad 5$ & - & 48 & 0 & 48 & 3 \\
\hline - & -7 & - & 48 & 4 & 48 & 9 \\
\hline - & - & Silberwiese & 48 & 4 & 48 & 7 \\
\hline - & -2 & $2 \quad-$ & 48 & 6 & 48 & 21 \\
\hline - & -1 & 1 Nagyag & 48 & 9 & 48 & 3 \\
\hline - & -3 & $3 \quad-$ & 48 & 5 & 48 & 7 \\
\hline
\end{tabular}
wenn auch kleiner Unterschiede in der Zone [cnbn'c] zwischen den Winkeln $n: b$ und $n^{\prime}: b$.

Nach sorgfältigen Messungen dieser Winkel gewinnt man die Ueberzeugung, dass zwar Differenzen von beträchtlicher Grösse auftreten, in denselben sich jedoch keinerlei Regelmässigkeit erkennen lässt. In Nachslehendem sind die Messungen an zebn der besten Krystalle aufgefuhrt: 


\begin{tabular}{ccccccc} 
& & \multicolumn{3}{c}{$n: b$} & \multicolumn{3}{c}{$n^{\prime}: b$} \\
Krystall Nr. 7 Nagyag & $48^{0}$ & $7^{\prime}$ & & $48^{0}$ & $8^{\prime}$ \\
& & & 48 & 1 & 48 & 3 \\
- & -8 & - & 48 & 3 & 48 & 18 \\
& & & 48 & 12 & 48 & 13
\end{tabular}

Diese Messungen zeigen Unterschiede von $001^{\prime}$ bis $0015^{\prime}$ zwischen den entsprechenden Winkeln, während die Abweichungen von dem für das rhombische System berechneten Werthe $48^{0} 7^{\prime}$ sich zwischen $0^{0} 0^{\prime}$ und $0^{0} 14^{\prime}$ bewegen; jedoch giebt sich, wie schon erwähnt, keinerlei Gleichmässigkeit zu erkennen. In einigen Fällen sind beide Winkel grösser, in anderen kleiner und in wieder anderen ist der eine grösser, der andere kleiner als der theoretische Werth. In zwei Făllen (Silberwiese Krystall 2, Nagyag Krystall 8) ist die Abweichung einer einzelnen Fläche eine beträchlliche, in anderen Fällen (Nagyag Krystall 7 und 8, Přibram Krystall 5) sind die Schwankungen des Winkelwerthes beider Flächen mit $b$ beträchtlicher, was auf eine Variation der Krystallelemente hinzuweisen scheint ${ }^{1}$ ). Was die in dieser Zone auftretenden Flächen anbetrifft, so erseheint gewöhnlich nur die Form $n$. Wo noch andere Formen in dieser Zone erscheinen (siebe Fig. 6), z.B. $k$, in der gewöhnlichen Stellung $\{013\}$, oder $\Sigma$, früher $\{031\}$, ist die Ausbildung vorn und hinten dieselbe und ergiebt sich auch die gleiche Uebereinslimmung der Winkel zu beiden Seiten von $b$, z. B. an Krystall 2 von Silberwiese $k: b=7^{0} 23^{\prime}, k^{\prime}: b=7^{0} 27^{\prime}, \Sigma: b=$ $20^{\circ} 29^{\prime}, \Sigma^{\prime}: b=20^{0} 27^{\prime}$.

Soweit die Messungen und die Flächenvertheilung eine Bestimmung gestatten, herrscht also in der betrachteten Zone rhombische Symmetrie. Gewisse Unregelmässigkeiten im Auftreten der Flächen in der bisherigen Prismenzone [m.m] dagegen fubrten zu der Ansicht, dass vielleicht bei Annahme der bisherigen Basis $c$, der gewöhnlich vorherrschenden Fläche, als einzigen Symmetrieebene $\{010\}$, eine monokline Symmetrie zu erkennen wäre. Alsdann müsste der Winkel der beiden Pinakoide $a\{100\}$ und $b\{010\}$ eine Abweichung von $90^{\circ}$ zeigen. Ganz tadellos ausgebildet waren beide Flächen nur bei einem von den gemessenen Krystallen. Dieser ergab $a: b=90^{\circ} \iota^{\prime}$. Die Abweichung lag also innerhalb der Fehlergrenze. Ausserdem erwies sich der Winkel $a: b$ bei Messung in verschiedener Temperatur als constant. Die Messungen an dem eben erwähnten Krystalle, ausgefuhrt mit dem grossen Fuess'schen Goniometer Nr. 1 unter viermaligen Repetitionen, ergaben :

1) Zirkel, Silzungsber. d. k. k. Akad. Wien 44, 447-448. - Miers, Mineral. Magazine 1884, 6, 65. 


$\begin{array}{cc}\text { Temperatur. } & a(100): b(010) . \\ 22{ }^{0} \mathrm{C} . & 90^{0} \mathcal{1}^{\prime} 23^{\prime \prime} \\ 110 & 90122 \\ 120 & 90120 \\ 120 & 90128 \\ 132 & 90122 \\ 144 & 90127 \\ 144 & 90117 \\ 22 & 90124 \\ 22 & 90116\end{array}$

Bei der zuletzt erwähnten Aufstellung müssten in der Zone $\left[a m b m^{\prime} a\right]$ constante, wenn auch kleine, Verschiedenheiten zwischen den Winkeln $m: b$ und $m^{\prime}: b$ vorhanden sein. An dem Krystall, welcher die beste Messung für $a: b$ gab, war nur eine $m$-Fläche ausgebildet. An allen anderen war die Fläche $b$ nicht so vollkommen, um einen so kleinen Unterschied jener beiden Winkel sicher zu stellen. Wie folgende Zahlen erkennen lassen, zeigen daher die beiden Winkel erhebliche und ganz unregelmässige Schwankungen:

\begin{tabular}{|c|c|c|c|c|c|}
\hline Pr̈ibram & Krystali & 6 & $46^{0} 59^{\prime}$ & $46^{\circ}$ & $045^{\prime}$ \\
\hline- & - & 12 & 4655 & 47 & 2 \\
\hline Silberwiese & Krystall & 2 & 475 & 46 & 52 \\
\hline Offenbanya & Krystall & 1 & 4629 & 46 & 48 \\
\hline- & - & & $46 \quad 42$ & 46 & 42 \\
\hline Nagyag & Krystall & 1 & 4656 & 46 & 49 \\
\hline- & - & 3 & 4651 & 46 & 47 \\
\hline- & - & 4 & 4656 & 46 & 57 \\
\hline - & - & 5 & 4644 & 46 & 47 \\
\hline
\end{tabular}

Was nun die ubrigen abgeleiteten Prismen $f$ und $e$ betrifft, so zeigt sich haufig eine Verschiedenheit zu beiden Seiten von $b$, doch liegt, wie weiterhin gezeigt werden soll, dieser unsymmetrischen Flächenvertheilung keinerlei Gesetzmässigkeit zu Grunde, und ebenso wenig konnte ein Unterschied beider Flächen $m$ und $m^{\prime}$ in Bezug auf die Häufigkeit der nach ibnen stattfindenden Zwillingsbildung nachgewiesęn werden. Endlich zeigt auch die Untersuchung der Wärmeleilung (siehe Anhang) vollständige rhombische Symmetrie auf der Fläche $c$, so dass die zuletzt angenommene monokline Stellung - in welcher ubrigens das krystallographische Axenverhältniss weniger gut mit dem der Feuerblende ubereinstimmt, als in der ersten Stellung - ebenfalls sich als unstatthaft erwiesen hat.

Zur Begrundung Dieses möge im Folgenden eine kurze Beschreibung der aäher untersuchten Krystalle mitgetheilt werden, weil sich hierbei auch die Gelegenbeit bietet, die bisherigen Angaben uber die Eigenthum- 
lichkeiten des Habitus der Krystalle mehrerer Fundorte zu vervollständigen. Hierbei ist die gewöhnliche Stellung der Krystalle beibebalten, bei welcher die an den tafeligen Bournonitkrystallen vorherrschende Fläche $c$ das Zeichen $\{001\}$ erhält, und zwar sind alle Buchstabenbezeichnungen und Symbole ubereinstimmend mit dem Referat uber Miers' citirle Arbeit in dieser Zeitschrift 11, 175.

Die Abbildungen sind zum grössten Theile, der Deutlichkeit wegen, Projectionen, in denen der Krystall mit der Axe $b$ aufrecht gestellt ist, während $a$ von rechts nach links, $c$ von vorn nach hinten verläuft.

In Zwillingen resp. Drillingen sind die Individuen mit I, II und III bezeichnet und zwar so, dass mit I das am grössten ausgebildete, mit II das mehr zurucktretende Individuum u. s. w. bezeichnet ist.

Der Kürze und Bequemlichkeit halber sei die Lage und das Individuum, zu welchem eine bestimmte Fläche gehört, durch Accente angedeutet, z. B. in Fig. 10 (Drilling) sei von den beiden Makropinakoidflächen, welche zum Individuum I gehören, die rechte mit $a$ ', die linke mit ' $a$ bezeichnet; von den beiden demselben Individuum angehörenden Prismenflächen ist die eine obere rechte mit $m^{\prime}$ und die obere linke mit ' $m$ bezeichnet. Flächen, welche zum Individuum II oder III gehören, haben zwei resp. drei Accente, es bezieht sich also " $b$ auf das linke Brachypinakoid von II, und "'b und $b^{\prime \prime \prime}$ auf die beiden Brachypinakoidflächen von III. Die eine (rechte) gehört zum lamellaren, die andere (linke) zum Haupttheil des Individuums.

\section{Prribram.}

Die Krystalle dieser Localităt können nach der Art ibrer Zwillingsbildung in drei Typen eingetheilt werden.

1. In Krystalle, welche als einfache bezeichnet werden können und nur von einer oder mehreren Zwillingslamellen parallel einer einzigen Fläche $(110)$ oder $(1 T 0)$ durchsetzt werden.

2. In Krystalle, welche aus zwei wohlentwickelten Zwillingsindividuen parallel $(110)$ oder $(\overline{1} 10)$ bestehen.

3. In Krystalle, welche sich aus drei Individuen (Drillinge) nach (110) und (1T0) oder (110) und (T10) zusammensetzen.

Von den zu diesen drei Typen gehörigen Krystallen brauchen wir vur die unter 1. und 3. näher in Betracht zu ziehen.

Der erste Typus der Krystalle dieses Fundortes ist sebr gut im Krystalle Nr. 2 (Fig. 1) ${ }^{1}$ ) ausgebildet. Dieser ist hinsichtlich der Vertheilung seiner Flächen von auscheinend monoklinem Habitus. Es erscheinen in

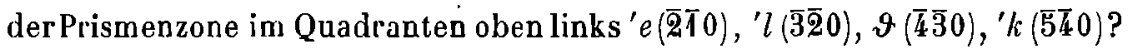

\footnotetext{
1) Original in der Mineralog. Slaatssamml. zu München.
} 
und ' $m(\bar{T} T 0)$, von denen ' 9 , ' $l$ und ' $m$ klein ausgebildet sind. Zu allen diesen Flächen waren auch die parallelen unten rechts vorhanden mit Ausnahme von $k_{,}\left(\overline{5} \bar{L}_{0}\right)$, welche zweifelhaft ist, wäbrend oben rechts nur $m^{\prime}(\overline{1} 10)$ erscheint. Unten links isl der Krystall zerbrochen. Die Messungen ergaben:

$\begin{array}{rlrl}\text { Beobachtet: } & & \text { Berechnet: } \\ 9: a=34^{0} \mathbf{5 4}^{\prime} & 35^{0} \mathbf{8}^{\prime} \\ l: a=3154 & 321 \\ e: a=2524 & 258 \\ k: a=3728 & 3653 \\ m: a=4626 & 4650\end{array}$

Die Pyramiden $u\{112\}$ und $y\{111\}$ erscheinen in allen drei erhaltenen Quadranten, sowohl oben wie unten; $\varrho\{121\}$ erscheint oben und unten in den beiden rechten Quadranten, feblt aber oben zur Linken. Der Krystall ist an diesem Theile etwas beschädigt und die Flächen scheinen weggesprengt zu sein; dasselbe trifft auch in Bezug auf die Flächen ' $\mu(\overline{3} \overline{3} 2)$ und ' $v(\overline{2} T 1)$ an der oberen Seite im selben Quadranten zu. Von den beiden letzterwähnten Flächen hat $v(\overline{2} T 1)$ die ihr correspondirenden in rechtsseitigen unteren Quadranten oben und unten, während $\mu(\overline{332})$ fehlt. $\odot(213)$ tritt ebenfalls in dem oberen linken Quadranten (oben und unten) und in dem unteren rechten Quadranten auf. Diese beiden beträchtlich formenreichen Quadranten sind gleichzeitig durchsetzt von zwei oder drei sehr dünnen Zwillingslamellen, und dass solche gewissermassen Flächen induciren, wurde mehrfach beobachtet. Dieser durchgehende grössere Flächenreichthum an einer Zwillingslamelle fuhrt dazu, dass die Krystallprojectionen oft jene ungleichförmige Flächenvertheilung aufweisen, die dem Krystall ein deutlich monoklines Aussehen giebt.

Ein weiteres Beispiel dieser Klasse liefert Krystall Nr. 6 (Fig. 2)1). Die Projection zeigt einige Unregelmässigkeiten in der Vertbeilung der Flächen und eine ähnliche Tendenz zu monokliner Symmetrie wie Krystall Nr. 2 (Fig. 1).

Krystalle des dritten Habitus von Přibram zerfallen in zwei Untertypen :

a) jene mit zwei wohlentwickelten Individuen und

b) solche, in denen eines der Individuen oder zuweilen beide auf Lamellen reducirt sind, die ein einziges Hauptindividuum durchsetzen.

Krystall Nr. 8 (Fig. 3) ${ }^{2}$ ) illustrirt den Subtypus a) dieser Klasse. Derselbe besteht aus Individuen von nahezu gleicher Entwickelung und ist nach (110) und (1T0) verzwillingt. Der Krystall ist besonders reich an

1) Original in der mineral. Sammlang d. deutschen Universilăt in Prag.

2) Original ebendaselbst. 
Pyramiden, welche, wenn auch nicht in gleicher Entwickelung, an allen drei Individuen erscheinen. Die Pyramiden $\varphi\{113\}, u\{112\}, y\{111\}$, $\Theta\{17.17 .12\}, \odot\{213\}$ und $v\{211\}$, welche als wohlentwickelte Flächen links von I auftreten, fehlen zur Rechten desselben Individuums vollständig. An II erscheinen vier derselben, wenn gleich nur spärlich entwickelt, nämlich: $\varphi\{113\}, u\{112\}, y\{111\}$ und $\Theta\{17.17 .12\},\left(\Theta: m=27054^{\prime}\right.$, berechnet $\left.=28^{1} 8^{\prime}\right)$. Der Krystall ist an der hinteren Seite schlecht entwickelt. Die Prismenzone zeigt einige leichte Unregelmässigkeiten, indem $e\{210\}$ an I nur mit einer einzigen Fläche ' $e(\overline{2} \bar{T} 0)$ erscheint; im Ganzen indessen ist die Vertheilung der Flächen eine sehr regelmässige.

Abbildungen des Subtypus b) zeigen die Fig. 4 und 5, Krystalle Nr. 11 und 12. Von diesen zeigt der erste (Krystall Nr. 11) ${ }^{1}$ ) einen Mangel an Symmetrie, der verursacht wird durch das Dazwischentreten von III, welches jenen Theil des Individuums I ersetzt, an welchem die Flächen $T^{\prime}(\overline{3} 21)$ und $v^{\prime}(\overline{2} 11)$ auftreten sollten. Andererseits wird das Erscheinen von $\varrho\left(T_{\overline{2}} 1\right)$ durch II verhindert. Die Unterseite des Krystalles konnte nicht untersucht werden, da derselbe hier auf einem Aggregat von Siderit, Zinkblende und Pyrit aufsitzt.

$\begin{array}{rlrl} & \text { Beobachtet: } & \text { Berechnet: } \\ k^{\prime}(\overline{5} \overline{4} 0): a(T 00)=36^{0} 47^{\prime} & 36^{0} 53^{\prime} \\ T^{\prime}(\overline{3} 21): o^{\prime}\left(T_{01}\right)=3952 & 3956 \\ v^{\prime}(\overline{2} 11): o^{\prime}(\bar{T} 01)=28 \text { 22 } & 2859 \\ { }^{\prime} \rho(\bar{T} \overline{2} 1):{ }^{\prime} y(T T 1)=1850 & 1924\end{array}$

Der andere, in Fig. 5 abgebildete Krystall weist eine sebr regelmässige Entwickelung auf, besonders in der Prismenzone, in welcher vollständige rhombische Symmetrie herrscht. Auffallend ist das Fehlen der gewöhnlichen Flächen des Brachydomas $n\{011\}$.

Beide Krystalle illustriren die bei Bournonit gewöhnliche Thatsache, dass, wenn $m\{110\}$ oder $a\{100\}$ von einer, wenngleich noch so dunnen, Zwillingslamelle durchsetzt werden, beide Antheile eine Verschiedenheil zeigen, indem entweder der eine gegen den anderen im Wachsthum zuruckgeblieben ist, was sehr häufig vorkommt, oder, im Falle eines gleichmässigen Wachsthums, indem, durch einige der häufigeren Flächen begrenzt, ein einspringender Winkel gebildet wird. Die Lamelle, welche diese Sonderung hervorruft, kann entweder in Verbindung mit einer der Lamellen fortgewachsen oder, falls sie nur schwach ausgebildet ist, in der Entwickelung zuruckgeblieben sein. Häufig kommt es vor, dass eine Heihe stark entwickelter Zwillingslamellen von einer Reihe schwach ausgebildeter, mehr oder minder von einander abstehender Zwillingslamellen, die gegen die

1) Original in der Sammlung der k. k. Bergakademie zu Přibram.

Groth. Zeitschrift f. Krystallogr. XXVII. 
ersteren eine Neigung von $93^{\circ} 40^{\prime}$ aufweisen, begleitet sind. Da wo diese Lamellen die Prismenzone schneiden, wird der Hauptkrystall in eine Reihe paralleler Gruppen zertheilt (wie in Fig. 1), während die andere Hälfte des Krystalles aus nicht-parallelen (in Zwillingsstellung befindlichen) Theilen zusammengesetzt ist.

\section{Silberwiese bei Ems.}

Die drei untersuchten Krystalle dieses Fundortes $\left.{ }^{1}\right)$ sind wegen ihres Flächenreichthums bemerkenswerth. Nr. 1 und 2 (Fig. 6) sind einfache, von einer $Z$ willingslamelle durchschnittene Krystalle. Nr. 3 (Fig. 7) ist ein Zwilling, der aus zwei gut ausgebildeten Individuen besteht.

Krystall Nr. 2 (Fig. 6) verdient wegen der grossen Zahl von Domenflächen, welche derselbe aufweist, erwähnt zu werden. Drei Makro- und drei Brachydomen sind leicht zu unterscheiden, wäbrend schwache Reflexe noch auf weitere drei der Makroreihe angehörige Domen hinweisen.

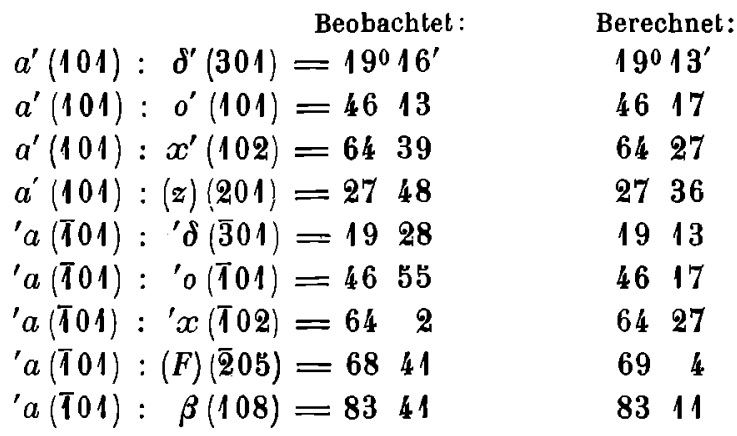

Mit Ausnahme des Auftretens der isolirten und nur schwach entwickelten Flächen $\varphi(1 \bar{T} 3)$ und $\odot(2 \bar{T} 3)$ sowie $e(2 T 0)$ und $\vartheta(430)$, ist die Vertheilung eine ungewöhnlich symmetrische. Die von Miers beobachtete, aber von ihm als zweifelhaft angesehene Form \{132\} erscheint als ein schmaler, in der Zone $[m \varrho n]$ und $[b u x]$ liegendes Flächenpaar mit folgenden Winkeln :

$$
\begin{aligned}
& (132)^{\prime}: m^{\prime}(110)=43^{0} 28^{\prime} \\
& \left.{ }^{\prime}(\overline{1} 32): ' m(\overline{1} 10)=4320 \quad \text { (berechnet } 43^{016} 6^{\prime}\right) \\
& (1 \overline{3} 2)^{\prime}: b^{\prime}(010)=3933 \\
& { }^{\prime}(\overline{1} 32): b^{\prime}(010)=3932 \quad\left(\text { berechnet } 39029^{\prime}\right) .
\end{aligned}
$$

Die correspondirenden Flächen an der Rückseite sind, obgleich schwach ausgebildet, noch sichtbar.

Krystall Nr. 3 (Fig. 7) scheint, obgleich nicht so flächenreich, ein neues Brachydoma $\dot{\varepsilon}$ aufzuweisen. Dasselbe erscheint mit einer wohlausgebildeten

1) Diese Krystalle stammen aus der Sammlung des Böhmischen Nationalmuseums zu Prag. 
Fläche zwischen $o^{\prime}(T 01)$ und $c(001)$. Zwischen demselben und $u^{\prime}\left(T_{12}\right)$ tritt eine scharf ausgebildete Fläche von $\xi^{\prime}(\overline{2} 14)$ auf. $\dot{\varepsilon}$ liegt, wie aus der Combinationskante mit $\xi^{\prime}$ hervorgeht, nicht in derselben Zone mit $\xi^{\prime}$ und $u^{\prime}$, sondern gehört vielmehr einem Doma mit kleinerer c-Axe an. Der Reflex der in Rede stehenden Fläche ist gut und hat eine maximale Fehlergrenze von $0^{0} 12^{\prime}$; der Reflex der unmittelbar unter ihr liegenden Fläche ó (T01) ist ausgezeichnet. Der Winkel zwischen beiden Flächen beträgt $24^{\circ} \mathbf{5 5}$ '. Hiernach fällt die Flăche zwischen

$$
\begin{aligned}
& F^{\prime}(205)\left(F: 0=22047^{\prime} \text { berechnet }\right) \text { und } \\
& \varepsilon(103)(\varepsilon: 0=26 \text { 2 berechnet }) \text {. }
\end{aligned}
$$

Fưr das allerdings complicirte Zeichen $\{9.0 .25\}$ ist der berechnete Winkel $i: 0=24^{0} 44^{\prime}$. Die Fläche ist zu gut beschaffen und die Reflexbilder derselben zu scharf, als dass ein Fehler von mehr als $0012^{\prime}$ angenommen werden könnte, sonst wäre dieselbe vielleicht als eine unvollkommen entwickelte Flache $\varepsilon(103)$ anzusehen.

\section{Horhansen.}

Die Krystalle dieser Localităt lassen sich in drei Typen unterscheiden:

1) Zwillinge nach einer einzigen Prismenfläche.

2) Solche nach zwei Prismenflächen (110) und (1T0), d. h. Drillinge, deren Brachydiagonalen im Mittelpunkte zusammentreffen, und deren Makropinakoide nach aussen gericbtet sind und mit einander Winkel von $9^{0} 40^{\prime}$ bilden.

3) Zwillinge nach zwei Prismenflächen (1T0) und (TT0), d. h. Drillinge mit im Centrum zusammentreffenden Makrodiagonalen und nach aussen gekehrten Brachypinakoiden, deren Winkel $86^{\circ} 20^{\prime}$ betragen.

Die Krystalle des ersteren Typus, anscheinend die gewöhnlichsten, bedürfen keiner weiteren Erwähnung. Dagegen bieten gewisse Krystalle der zweiten und dritten Typus Einiges, das unser Interesse beansprucht.

Nr. 1 (Fig. 8) ${ }^{1}$ ) kann als Repräsentant der Krystalle des zwoiten Typus dieser Localität gelten. Obgleich der Krystall wegen seiner Grösse und Flächenbeschaffenheit keine sehr genauen Messungen zuliess, so genügten diejenigen der Prismenzone doch, die in der Zeichnung wiedergegebene Anordnung der Flächen sicher zu stellen. Man bemerkt, dass die Theile I stets an der rechten Seite jeder der feinen eingelagerten Zwillingslamellen, welche mit einer annähernd $f\{120\}$ des Krystalls II entsprechenden Fläche endigen, vorspringen. Zweitens fällt eine ungleichmässige Vertheilung der Flächen auf, indem $e\{210\}$ und $f\{120\}$ nur rechts und $e\{210\}$ und $l\{320\}$ nur links auftreten.

1) Original in der Privatsammlung des Herrn Seligma n $\mathbf{n}$ in Coblenz. 
Der Krystall Nr.5(Fig. 9) ${ }^{1}$ ) kann als ein Muster der Krystalle des drilten Typus dieser Localität gelten. Derselbe besteht aus einem Individuum I, an welches sich links und rechts, mit den Flächen (1T0) und (TT0) verwachsen, die Individuen II und III anlegen, deren jedes von einer zu I gehörigen Zwillingslamelle, parallel den beiden genannten Ebenen, durchsetzt wird; infolgedessen bilden diese beiden Antheile des Individuums I ein V. Der Zwillingswinkel I und II ergab sich $0035^{\prime}$ grösser, der zwi- schen II und III $108^{\prime}$ geringer als der theoretische Werth, während die Winkel zwischen ' $b(010)$ und $a(100)=90^{\circ} 2^{\prime}$ und $b^{\prime}(010)$ und $a(\bar{T} 00)$ $=89^{\circ} 58^{\prime}$ sehr gut unter einander und mit dem theoretischen $\left(90^{\circ} 0^{\prime}\right)$ übereinslimmen. Auffallend ist die ungleichmässige Entwickelung der zum Theil I und III gehörigen Pyramiden zur Rechten im Vergleich mit jenen zur Linken.

Eine Messung der Zone, in welcher die ersteren liegen, zeigt, dass sie der Form $\varphi$ (113), $u(112)$ und $y(111)$ angebören; biervon sind $\varphi(113)$ und $u$ (112) gut entwickelt, und $y(111)$ durch $f^{\prime}(120)$ zurüuckgedrängt; links dagegen erscheint $u$ (112) sehr gross und $y$ (111) sehr schmal, während $\varphi$ (113) gänzlich fehlt. Es düfte sich hierin schwerlich mehr als eine zufăllige Unregelmässigkeit in der Flächenentwickelung ausdrücken. Eine Gesetzmässigkeit ist darin nicht zu finden, wie aus der Thatsache hervorgeht, dass $\varphi(11 \overline{3})$ an der Rückseite von I ebenfalls fehlt. Dazu kommt noch, dass diese unregelmässige Vertheilung der Pyramiden ausgeglichen wird durch die absolut symmetrische Entwickelung der Flächen in der Prismenzone. Es würde noch die ungewöhnliche Entwickelung von ' $f$ (120) und $f^{\prime}(120)$ zu erwähnen sein; dieselben sind sebr gross, nehmen einen Umfang an, wie er gewöhnlich nur dem primären Prisma zukommt, verdrängen in diesem Falle ' $m(T 10)$ und $m^{\prime}(110)$ völlig und kommen direct rechts mit $m^{\prime \prime \prime}(\overline{1} 10)$ und links " $m(110)$ in Bertubrung. Mit jener bilden sie einen Winkel von $10^{\circ} 58^{\prime}$ und mit dieser einen solchen von $11^{\circ} 32^{\prime}$ (theoret. 1 1026' oder $\left.f^{\prime}(120): m^{\prime}(110)=18^{0} 46^{\prime}-70^{\circ} 0^{\prime}\right)$. Hierdurch wird ein weiterer Beweis erbracht für die beträchtliche Differenz zwischen den Zwillingswinkeln zur Rechten und Linken; der eine zwischen I und III ist $0^{0} \mathbf{2 8}^{\prime}$ kleiner, der andere zwischen I und II $0^{0} 6^{\prime}$ grösser als der theoretische Werth. Die Reflexe aller dieser vier Flächen sind gut.

\section{Offenbanya.}

Der einzige Repräsentant dieses Fundortes, ein wenig vollkommener Krystall, dessen Flächen alle mehr oder minder matt waren, ist in Fig. 10 auf Grund der Messungen der Prismenzone dargestellt ${ }^{2}$ ). Von den drei In-

1) Original im Hofmineraliencabinet in Wien.

2) Das Original stammt aus der Mineraliensammlung des Böhmischen Nationalmuseums in Prag. 
dividuen desselben ist II mit I nach einer Fläche (TT0) verwachsen. Es wird durchschnitten von einer sehr dunnen Lamelle. III besteht aus einer Hauptpartie und einer lamellaren Fortsetzung; letztere ist parallel der Zwillingsebene (1T0), während erstere mit einer Fläche senkrecht dazu mit I verwachsen, und II in einer Fläche ungefähr parallel $m_{\prime \prime}(\bar{T} T 0)$ berührt. Der Haupttheil von III zeigt eine sehr unvollkommene Fläche, die innerhalb $1^{0} 11^{\prime}$ mit $\Psi(\breve{\jmath} \overline{6} 0)$ tbereinstimmt und mit , $m(T 10)$ einen Winkel von $0^{0} 33^{\prime}$ bildet. Der lamellare Theil trägt eine Fläche $f_{\prime \prime \prime}(120)$, mittels welcher derselbe im Verein mit $m^{\prime}(110)$ an der äusseren Peripherie des Krystalles unten eine ausgesprochene Sonderung von I bewerkstelligt, während derselbe oben eng mit letzterem verwachsen ist. Diese ziemlich ungewöhnliche Entwickelung eines einzigen Individuums, wie sie III darbietet, wurde auch an Krystallen von Nagyag constatirt ${ }^{1}$ ).

\section{Nagyag.}

Die Bournonitkrystalle von dieser Localität sind von T. A. Quens tedt in seinem Handbuch der Min. S. 890 als Durchkreuzungszwillinge beschrieben worden, die denen des Cerussit völlig analog sind und im Aussehen denen des Staurolith ähneln. Ferner beschrieb G. vo m R ath ${ }^{2}$ ) eine einzelne interessante Krystallgruppe dieses Fundortes. Dieselbe besteht aus vier Individuen mit nach einem Centrum laufenden Makrodiagonalen und nach aussen gekehrten Brachypinakoiden, welche mit einander die Winkel $79^{\circ} 59^{\prime}$, 920 $28^{\prime}, 96^{\circ} 48^{\prime}$ und $90^{\circ} 44 \frac{1}{2}^{\prime}$ bilden. Diese Winkel beweisen nach der Angabe des genannten Forschers, dass die Krystalle nicht in Zwillingsstellung zu einander stehen können. Einer derselben $\left(\mathbf{9 2}^{0} \mathbf{2 8}^{\prime}\right)$ liegt nahe dem Zwillingswinkel des Bournonit. In Bezug auf diesen schrieb er : "Die Individuen I und II legen allerdings annähernd eine Prismenfläche $m$ (als $m^{\prime}$ bezeichnet) in gleiches Niveau; man könnte geneigt sein, sie als Zwillinge - verwachsen mit einer Fläche normal zur Zwillingsebene - zu betrachten. Dieser Deutung wurde indess der Winkel

1) Derartige unregelmässige Formen der Zwillingspartien kommen auch an anderen Fundorten vor, wie z. B. der in Fig. 11 abgebildete Krystall zeigt. Dieser ungewöhnlich grosse Krystall von Meiseberg bei Neudorf am Harz (Original im Hofmineraliencabinet in Wien, Cat.-Nr. 176) hat etwa $5 \mathrm{~cm}$ Durchmesser und zeigt eine breite Bruchflàche annähernd parallel \{001\}. Auf dieser Bruchfläche kann man deutlich die Grenze von vier Individuen verfolgen. Der Haupttheil des Krystalles besteht aus I, welches von den das Individuum II bildenden breiten Lamellen durchschnitten wird. Die erste zu II gehörige Lamelle besteht aus einer unregelmässigen, von I, III und IV umschlossenen Masse mit einer lamellaren Fortsetzung. Die erste zu I gehörige Lamelle ist gleichfalls ganz unregelmässig und nahezu von I und IV umschlossen. III und IV sind verhältnissmässig kleine, unregelmăssige Massen; die letztere ist wieder ein zusammengesetztes Individuum.

2) Diese Zeitschr. 1877, 1, 602 . 
$92^{0} 28^{\prime}$ entgegen stehen, welcher mehr als $1^{0}$ von der Zwillingskante des Bournonit $\left(93^{\circ} 40^{\prime}\right)$ abweicht. *

Was der eben genannte Forscher aus der Stellung der Krystalle entnahm, ist durch die Untersuchung bestätigt worden, die an dem aus diesem Fundorte stammenden Material vorgenommen wurde.

Die Krystalle ${ }^{1}$ ) sind von zwei Typen :

1) Zwillinge mit gemeinsamer Zwillings- und Verwachsungsfläche, einfache Formen.

2) Zwillinge und Drillinge, deren Verwachsungsfläche rechtwinkelig zur Zwillingsebene steht, complicirtere Formen.

Krystall Nr. 5 wurde als Repräsentant des ersteren Typus gewählt. Die Messungsergebnisse führten zu der in Fig. 12 angegebenen Deutung der Flächen. Dieser Krystall zeigt, wie die Krystallbildung vorwiegend nach einer einzigen Fläche (hier (110) von I) vor sich gehen kann. Dieselbe kommt in dieser Form an Krystallen von Pribram und Horhausen vor. Der vorliegende. Krystall ist theilweise bedeckt von Manganblende, und die Flächen sind nicht so glänzend, wie bei den anderen Krystallen dieses Fundortes. Derselbe besteht aus vier Lamellen, welche zu I gehören und die durch drei zu II gehörige Lamellen getrennt werden. Von den vier zu I gehörenden sind die beiden äusseren sehr schmal; bei der einen wird dies durch den zerbrochenen Zustand des Krystalles bedingt, während die andere von Natur aus unvollkommen entwickelt ist und den äussersten rechten Theil des Krystalles bildet. Die drei Theile des Brachypinakoides ' $b\left\{\begin{array}{lll}0 & 0 & 0\end{array}\right\}$ spiegeln zusammen und bilden mit den zwei Theilen des Makropinakoides $a^{\prime \prime}\{100\}$, welche gleichfalls zusammen reflectiren, einen Winkel von $3^{0} 19^{\prime}$ $\left(3^{0} 40^{\prime}\right)$. Die alternirenden Flächen $n^{\prime}\{011\}$ und $o^{\prime \prime}\{101\}$ berühren einander in Folge der Differenz der Winkel, welche sie mit $c\{001\}$ bilden $(n: c$ $\left.=41^{\circ} 53^{\prime} ; 0: c=43^{\circ} 43^{\prime}\right)$, in einer unregelmässigen Linie. Seine $u\{1 / 2\}-$ Flächen, welche in dieselbe Ebene fallen, erscheinen als eine einzige Fläche von schwach zickzackähnlicher Form ohne die Spur einer trennenden Linie. Die punktirten Linien in der Figur sind gezeichnet, um die Grenze der einzelnen Lamellen schärfer definiren zu können.

Die Krystalle Nr. 7 (Fig. 13), Nr. 8 und Nr.9 (Fig. 14) sind als Vertreter

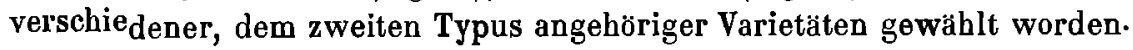

Von ihnen ist der erste (Fig. 13) der einfachste. Es ist ein Drilling, in welchem II mit I nach (1T0) verzwillingt und nach einer dazu senkrechten Fläche verwachsen ist. Dieses wird durch die folgenden Messungsresultate dargethan:

1) Sämmtliche Krystalle von diesem Fundorte stammen aus der Mineraliensammlung des Böhmischen Nationalmuseums in Prag. 


$$
\begin{aligned}
& b^{\prime}(010): b^{\prime \prime}(010)=93^{0} 14^{\prime}\left(93^{0} 40^{\prime}\right) \\
& a^{\prime}(100): a^{\prime \prime}(100)=8615(8620) \\
& u^{\prime}(112): u^{\prime \prime}(112)=017\left(\begin{array}{ll}
0 & 0
\end{array}\right)
\end{aligned}
$$

Individuum III von untergeordneter Bedeutung ist von schmaler, keilförmiger Gestalt und liegt zwischen I und II. Mit I steht es nach (1T0) in Zwischenstellung und beruhrt II an der Zusammensetzungsfläche zwischen diesem und $I$. Es endigt in einer schmalen Fläche $f_{\prime \prime \prime}(120)$, welche mit $" e(\overline{2} T 0)$ einen spitzen, einspringenden Winkel bildet, durch den es, zusammen mit I, von II getrennt wird. I ist charakterisirt durch die Grösse von ' $u\left(\right.$ T12) und $u^{\prime}(112)$, welche einander schneiden und $u^{\prime}(011)$ auf ein Dreieck beschränken. Sie werden ihrerseits in der Entwickelung durch die Makropinakoide $a^{\prime}(100)$ und ' $a($ T00) zuríckgedrängt und erhalten die Form unregelmässiger Sechsecke. $b^{\prime}(010)$ ist sebr schmal und schlecht spiegelnd. II zeigt das etwas ungewöhnliche Makrodoma $h\{208\}$ neben o $\{101\}\left(h^{\prime \prime}: a^{\prime \prime}=57047^{\prime}\right.$, berechnet 57029'). Es besteht aus zwei Theilen, êinem Haupttheil in Beruhrung mit I und III an deren Zusammensetzungsfäche, und einem keilförmigen Vorsprunge mit $f(1 \overline{2} 0)$, welcher in der Zwillingsebene mit $I$ in Berührung steht. I liegt in dem einspringenden rechten Winkel, der durch diese beiden Theile gebildet wird.

Krystall Nr. 8 ist ein etwas complicirteres Beispiel dieser Klasse. Die Flächen sind gut entwickelt und von demselben ausgezeichneten Glanze, wie bei dem oben beschriebenen Krystalle. Die breitere Basis giebt dem Krystalle ein tafelartiges Aussehen. Von den Domen ist nur das Brachydoma gut entwickelt, das Makrodoma fehlt, mit Ausnahme einer ganz untergeordneten Entwickelung zur Linken von I. Von den Pyramiden erscheint nur eine einzige $u\{112\}$, deren sämmtliche Flächen, mit einer einzigen Ausnahme, vom Makropinakoid geschnitten werden, wodurch sie eine dreieckige Gestalt erhalten. Beide Pinakoide haben eine gekörnte Oberfläche und geben nur schwache Reflexe. Dieselben sind wie beim ersten Beispiel sehr schmal und werden durch Brachydomen in der Entwickelung zuruckgedrängt. Prismen sind zwei vorhanden, $m\{110\}$ und $f\{120\}$, letzteres stark gestreift parallel der Axe $c$. Wie aus den Messungen hervorgeht, sind Individuum I und II mit einander verwachsen nach einer zur Zwillingsebene senkrechten Fläche. Ihre Beruhrungslinie verläuft als schwache, unregelmässige Linie auf der Basis. Die Prismenflächen $m^{\prime}(110)$ und $m^{\prime \prime}\left(T_{T} 0\right)$, welche in eine Ebene fallen sollten, bilden einen Winkel von $0^{0} 19^{\prime}$ mit einander, ebenso $u^{\prime}(112)$ und $u^{\prime \prime}\left(T_{T} / 2\right)$ einen solchen von 0012'. Die erstere Abweichung wird zum Theil durch die unvollkommene Ausbildung der $m$ Flăchen erklärt, die letztere entspricht einer geringfugigen Drehung um die Zwillingsaxe. Von den Zwillingswinkeln zwischen den beiden Pinakoidflächenpaaren ist jener zwischen dem Brachypinakoid $b^{\prime}$ und $b^{\prime \prime} 93^{\circ} 37^{\prime}$ mit dem theoretischen $\left(93^{\circ} 40^{\prime}\right)$ sehr nahe ubereinstimmend, während der 
zwischen den Makropinakoiden $a^{\prime}$ und $a^{\prime \prime}$, $86^{\circ} 43^{\prime}\left(86^{\circ} 20^{\prime}\right)$, dem rechten Winkel um $0^{0} 23^{\prime}$ zu nahe liegt. Auch hier sind beide Flächen unvollkommen, und die Winkel zwischen denselben und den resp. Brachypinakoiden wurden zusammen $0^{\circ} 23^{\prime}$ grösser als zwei Rechte gefunden $\left(b^{\prime}: a^{\prime}=90^{0} 10^{\prime}, b^{\prime \prime}: a^{\prime \prime}=90^{0} 13^{\prime}\right)$. I ist, soweit festgestellt werden kann, durchschnitten erstens von zwei zu II gehörigen Lamellen, welche parallel der gemeinsamen Zwillingsfläche liegen; eine Erweiterung eines Endes der ersten derselben bildet die Hauptmasse von II. Die beiden Zwischenlamellen, die zu I gehören, haben an ihrem rechten unteren Ende je eine $f\{120\}$-Fläche; in der That sind diese zwei Flächen gepaart, wie der Winkel zwischen denselben zeigt $\left(f,(1 \overline{2} 0): f(\overline{1} \overline{2} 0)=56^{0} 16^{\prime}\right.$, theoretisch $\left.56^{0} 8^{\prime}\right)$. Würden die Ebenen, in denen sie liegen, nach oben verlegt, so wurden diese beiden Flächen das entsprechende Flächenpaar, nämlich (120) und (120), bilden, d. h. je eine läge an jeder Seite von ' $b\{010\}$. Ferner wird I durchschnitten von einem zweiten Paar Lamellen, parallel (1T0), deren jede auch einen Theil derselben $f$-Fläche trägt, welche der Fläche des Krystalles I entspricht. Eine dieser Lamellen kann durch die feinen Grenzlinien auf der Basis bis an die zerbrochene Kante des Krystalles verfolgt werden. Ein wenig unter dieser Stelle ịst ein Krystallfragment zu seben; dieses ist deutlich von der letzten (vierten) Lamelle, welche zu I gehört, durch eine unregelmässige Linie getrennt, und trägt eine einzige, in der Prismenzone gelegene Fläche, die zusammen mit $m,\left(1 T_{0}\right)$ spiegelt und mit derselben einen einspringenden Winkel von $7032^{\prime}\left(7^{0} 20^{\prime}\right)$ mit $m_{\prime \prime}(110)$ und einen von $42^{\circ} 55^{\prime}\left(43^{0} 10^{\prime}\right)$ mit $a_{\text {, }}(100)$ bildet, woraus hervorgeht, dass dieser Theil des Krystalles der Rest eines Krystallindividuums III ist, welches mit I parallel (1T0) verzwillingt und unter rechtem Winkel dazu verwachsen ist. Ergänzen wir dieses Individuum zur Grösse der beiden anderen und nehmen noch ein viertes an, welches zum ersten dieselbe Beziehung wie II zu $\$ hat, so erhalten wir einen Vierling, welcher einem Durchkreuzungszwilling gleichen wurde, dessen vier Brachydomenflächen nach auswäts gekehrt wären. Unter den zwischen den letzteren liegenden Winkeln wären drei gleich $93040^{\prime}$, der vierte gleich $79^{\circ} 0^{\prime}$ oder $90^{\circ}-3\left(3^{\circ} 40^{\prime}\right)$. Die vier Messungen, welche vom Rath (l. c.) angiebt, nämlich $90^{\circ} 44^{\prime}, 96^{\circ} 48 \frac{1}{2}^{\prime}, 92^{\circ} 28^{\prime}$ und $79^{\circ} 59^{\prime}$, deuten gewiss auf einen ähnlichen Verband der vier von ihm beschriebenen Individuen.

Der dritte Reprăsentant dieses Typus (Fig. 14) liefert bessere Messungen als jeder der beiden anderen. In diesem Falle aber sind die Formen $u\{112\}$ und $m\{110\}$ die vorherrschenden, statt $n\{011\}$ und $b\{010\}$. Der Krystall besteht aus drei Individuen, wie in den beiden vorhergehenden Fällen. I bildet den Haupttheil des Krystalles, welcher von einer einzigen nachweisbaren Zwillingslamelle III parallel $\{1 \bar{T} 0\}$ durchschnitten ist. Wie in den zwei vorhergehenden Fällen endigt diese Lamelle mit einer Fläche von 
$f\{120\}$. Diese beiden Individuen I und III liegen, wie in dem zuerst angefuhrten Beispiele, in dem einspringenden rechten Winkel, welcher, durch die beiden Theile von II gebildet, zur Linken von der Zwillings- und zur Rechten von der Zusammensetzungsfläche begrenzt wird. Das Verhältniss der Individuen ist im vorliegenden Falle identiseh mit demjenigen des ersten, die hauptsächlichste Differenz zwischen beiden besteht in dem Unterschiede in der Grösse der Individuen I und II.

\section{Kapnik.}

Die Krystalle dieses Fundortes $\left.{ }^{1}\right)$ sind, soweit das spärliche, zur Verfugung stehende Material ubersehen lässt, meist complicirte Formen, im Wesentlichen indessen nahe übereinstimmend oder identisch mit den unter Typus II von Nagyag beschriebenen. Das Material ist zu Messungen nicht besonders geeignet. Die Flächen geben kaum in einem Falle gute Reflexe und sind oft so matt, rauh oder stark gestreift, dass sie uberbaupt kaum irgend einen Reflex mehr geben. Bei Verwachsungen senkrecht zur Zwillingsebene, oder bei parallelem Wachsthum sind ausserdem die Individuen oft so stark aus ihrer Lage gedreht, dass zwei zur selben Zone gehörige Flächen um einen Grad und mehr von derselben abweichen.

Wie aus den Messungen und der darnach angefertigten Fig. 15 ersichtlich, ist der Krystall Nr. 1 von dieser Localităt ganz ähnlich dem Krystall Nr. 7 (Fig. 13) von Nagyag. Individuum II ist hier mit I verzwillingt parallel (1T0) und rechtwinkelig dazu verwachsen. Es ergab sich:

$$
\begin{aligned}
& ' m\left(T(10): " m(T T 0)=0030^{\prime}\right. \\
& \prime a(T 00): " a(T 00)=8644 \\
& ' u(T T 2): " u(T 12)=0 \quad 15
\end{aligned}
$$

II zeigt Zwillingsbildung parallel (TT0), sowie eine Art des Wachsthums, durch welche $m^{\prime \prime}(110)$ in drei gesonderte Partien getheilt wird. Aehnliche Ausbildung ist an anderen Krystallen dieses Fundortes zu beobachten.

Fig. 16 (Krystall Nr. 2) zeigt einen Krystall, welcher ebenfalls auffallende Aehnlichkeit mit Typus 2 von Nagyag besitzt, wie ein Vergleich mit Fig. 14 lehrt. Thatsächlich repräsentiren Fig. 15 und Fig. 16 den Typus Nagyag ebenso gut wie Fig. 13 und Fig. 14. In Fig. 16 wie in Fig. 14 besteht der Krystall aus zwei vorherrschenden Individuen, II verzwillingt mit I nach (1T0) und damit unter rechtem Winkel dazu verwachsen, wie die, obgleich ungenauen, Messungen genügend erweisen:

$$
\begin{aligned}
& \text { ' } m(T 10): " m(\bar{T} T 0)=0055^{\prime}\left(\begin{array}{ll}
0^{0} & 0^{\prime}
\end{array}\right) \\
& \text { ' } a(T 00): a^{\prime \prime}(T 00)=8651\left(\begin{array}{ll}
86 & 20
\end{array}\right) \\
& a^{\prime}(100): a^{\prime \prime}(\bar{T} 00)=93 \quad 22(93 \quad 40)
\end{aligned}
$$

1) Sammtliches Material dieses Fundortes stammt aus der Privatsammlung des Herro Seligmann in Coblenz: 
I ist in II eingelagert genau wie bei Fig. 14 beschrieben, nur dass in diesem Falle I an Stelle eines dritten Individuums, das es durchdringt, von einer einzigen Lamelle von II durchsetzt wird. Auch ist jener Theil von II, der von I durch die Zwillingsfläche getrennt ist, anstatt enge mit ibm zusammenzuhängen, von ihm durch $e(210)$ und $a(100)$ getrennt. $b^{\prime}(010)$ und $n^{\prime}(011)$ werden durchschnitten von einer ausserordentlich dunnen Lamelle, welcbe mit der Kante zwischen ' $u$ (TT2) und $c(001)$ zusammenfällt, und durch die das Individuum $I$ in zwei parallele Partien getheilt wird.

Der Krystall, welchen Fig. 17 darstellt, ist von minderer Beschaffenheit. Er besteht aus drei Individuen, von welchen nach den Messungen II mit I nach $\left(1 T_{0}\right)$ in Zwillingsstellung zu stehen scheint und senkrecht dazu verwachsen ist.

$$
\begin{aligned}
& b(010): b^{\prime \prime}(010)=92^{\circ} 44^{\prime}\left(93^{\circ} 40^{\prime}\right) \\
& a^{\prime}(100): a^{\prime \prime}(100)=8732(8620) \\
& m^{\prime}(110): m^{\prime \prime}(110)=041\left(\begin{array}{ll}
0 & 0
\end{array}\right)
\end{aligned}
$$

II ist stark gedreht um die Zwillingsaxe, so zwar, dass die Reflexe von $a^{\prime \prime}$ und $b^{\prime \prime}$ erheblich aus der Prismenzone heraustreten. Dieses, im Verein mit der unvollkommenen Beschaffenheit der FJächen, ist wohl genugend, um diese Abweichung zu erklären. III füllt den Winkel zwischen I und II aus, obgleich es nicht in Zwillingsstellung mit denselben steht und auch nicht parallel zu II ist. Es ist indessen beinahe rechtwinkelig zu I $\left(b^{\prime}: b^{\prime \prime \prime}=\right.$ $\left.90044^{\prime}\right)$.

Die auffallende Aehnlichkeit der Krystalle von Nagyag im Vergleich mit jenen von Kapnik liess vermuthen, dass die Formen der ersten Localität als einfache und unvollständige Formen des Rädelerzes betrachtet werden könnten, von welchem verschiedene Erklärungen $\left.{ }^{1}\right)$ gegeben worden sind, und dass umgekehrt dem Rädelerz ein Vierling zu Grunde liege, wie wir ihn bereits beschrieben haben, bei welchem die einspringenden Winkel zwischen den Individuen von andern Krystallen eingenommen werden, entweder in Zwillingsstellung zu einem der Originalindividuen, wie beim Krystall von Offenbanya (Fig. 10, II), oder parallel zu einem derselben, oder endlich wie in Krystall Nr. 5 von Kapnik (Fig. 17, III), weder parallel noch in Zwillingsstellung zu demselben.

Wenn wir uns die ursprungliche Zwillingsbildung nach den vier Originalindividuen noch verwickelter denken durch das Auswachson der Prismenzone in zahnförmige Vorsprünge und die Bildung von einspringenden Winkeln, wie sie an den Krystallen Nr. 6, 9 und 12 (Fig. 2, 4 und 5) von Přribram zu seben ist, wenn wir uns ferner noch eine weitere Complication dadurch vorstellen, dass auch die secundären, in den einspringen-

1) Zirkel, Sitzungsber. k. k. Akad. Wien 44, 464. - Sa debeck, Angewend. Krystallogr. S. 120. - Ml iers, Mineral. Mag. 6, 74. 
den Räumen der ursprünglichen Form liegenden Individuen verzwillingt werden, so haben wir, so weit die vorliegende, allerdings begrenzte Untersuchung erkennen lässt, eine nicht unwahrscheinliche Erklärung dieser complicirten Formen des Bournonit.

Die folgenden Bemerkungen über den Bournonit von drei Localitäten, von welchen sich Proben in der Staatssammlung zu München befinden, mögen hier angeschlossen werden.

\section{Rosenhofer Zng bei Clausthal.}

Dieses Vorkommen wird von Zirkel erwähnt. Die Krystalle verdienen aber eine kurze Beschreibung wegen ihrer aufallenden Uebereinstimmung mit jenen von Liskeard. Es sind zum grössten Theile Zwillinge, bestehend aus zwei Individuen von gleicher Grösse, doch öfters complicirt durch parallele Wachsthumsgruppen. Gleichwie bei dem Typus Liskeard sind die Krystalle mehr oder minder von kubischer Form und zeigen eine Neigung zur unsymmetrischen Entwickelung der Flächen in der Prismenzone, indem $m\{1 T 0\}$ oft allein und gut entwickelt, jedoch nur an einer Seite erscheint, während an der anderen $e\{210\}, l\{320\}$ und $m\{110\}$ bäufig zu finden sind. Das Makrodoma $o\{101\}$ ist gewöhnlich gut entwickelt, während $a\{100\}$ auf eine schmale nahezu viereckige Fläche reducirt ist. Die Flächen sind zum grössten Theile glänzend.

Die Krystalle sitzen auf derbem Bournonit mit Braunspath und Baryt.

\section{Wolfsberg am Harz.}

Ein einziger grosser Krystall von diesem Fundorte scheint ein Drilling zu sein, bei welchem die Individuen parallel $c$ stark verlä́ngert sind. Der Krystall hat eine Länge von $2 \frac{1}{2} \mathrm{~cm}$. In der Prismenzone sind die drei den verschiedenen Individuen angehörige Pinakoidflächen vorherrschend, wodurch der Krystall ein pseudotetragonales Aussehen erhält. Ausser der Basis sind keine weiteren Flächen mit Sicherheit zu constatiren. Der Krystall bietel ein besonderes Interesse dadurch, dass in demselben zufolge einer Analyse eines Theiles desselben, welche Herr Prof. Muthmann ausführte, die Hälfte des Antimons durch Arsen ersetzt ist.

Er findet sich zusammen mit Quarz und Jamesonit.

\section{Bertha-Grube, Schwaz, Tirol.}

Die einzige mir bekannt gewordene Stufe dieses anscheinend wenig oder nicht bekannten Vorkommens befindet sich in der Münchener Sammlung. Die Krystalle sind klein und unvollkommen, von tafeliger Form und nach einer einzigen Prismenfläche verzwillingt. Die feststellbaren Formen sind $a\{100\}, o\{101\}, c\{001\}, b\{010\}, n\{011\}, u\{112\}$ und $y\{111\}$. Hin- 
sichtlich der Form gleichen diese Krystalle jenen von Horhausen. Sie finden sich auf einem Gemenge von derbem Fahlert und Siderit.

Am Schlusse dieser Arbeit sei es mir gestattet, meinen besten Dank denjenigen Herren auszudrucken, welche mir in liebenswürdigster Weise Material zu dieser Untersuchung iberlassen haben. Durch die Gute des Herrn Prof. Vrba in Prag kam ich in den Besitz von einigen ausgezeichneten Krystallen; ausserdem ermöglichte es mir Derselbe, das reiche und ausgezeichnete Material des Böhmischen Nationalmuseums für meine Zwecke zu benutzen. Herr Dr. A. Brezina in Wien überliess mir nicht nur bereitwilligst Material zu näherer Untersuchung, sondern ermöglichte es mir auch, das tuberreiche Material der Wiener Collection zu studiren. In ebenso liebenswürdiger Weise haben Herr Prof. Be cke in Prag und Herr Prof. Hof mann in Přibram mir Material zur eingehenderen Untersuchung geliehen und das Uebrige in den ihnen unterstellten Sammlungen zugänglich gemacht. Herrn G. Seligmann in Coblenz bin ich insbesondere verpflichtet für die durch lange Zeit gewährte Ueberlassung des vorzugglichen Materials seiner Privatsammlung. Meinen ganz speciellen Dank aber möchte ich Herrn Prof. Dr. P. G ro th, meinem hochgeehrten Lehrer, auf dessen Anregung ich diese Arbeit unternahm, aussprechen, nicht allein für die Ueberlassung des werthvollen Materials der Munchener Staatssammlung und fur die freundliche und entgegenkommende Unterstutzung, die er mir bei der Bestimmung der Methoden und Ziele der vorliegenden Arbeit gewährte, sondern ganz besonders für die im Allgemeinen von ihm empfangene, äusserst werthvolle Anleitung? und Belehrung auf dem Gesammtgebiete der Mineralogie und Krystallographie.

\section{Anhang.}

\section{Wärmeleitnng des Antimonit und Bournonit.}

Im Folgenden sind die Ergebnisse einiger Experimente uber die Wärmeleitung von Antimonit und Bournonit zusammengefasst. Die Versuche wurden vermittelst der von Prof. Dr. Röntgen in Würzburg erfundenen und in dieser Zeitschrift 3, 17 beschriebenen Methode ausgeführt.

Das erste der beiden genannten Mineralien eignet sich besonders $\mathrm{zu}$ Experimenten, bei denen diese Methode zur Anwendung gelangt, indem die aussergewöhnlich vollkommene Spaltbarkeit desselben nach $\{010\}$ zur Vornahme der Versuche ausgezeichnete Flächen bietet. Ausserdem bot sich hierbei die Gelegenheit die Frage zu prufen, ob die von Drude (s. diese Zeitschr. 18, 648) gefundenen Abweichungen gewisser optischer Eigenschaften des Antimonit von der rhombischen Symmetrie von Zufälligkeiten 
in der Beschaffenheit seines Materials abhängen oder nicht, d. h. ob sich auch eine Verschiedenheit der Orientirung der Axen des Wärmeleitungsellipsoides von derjenigen der krystallographischen Axen ergeben würde, in welchem Falle der Antimonit nicht dem rhombischen Systeme angehören könne.

Aus ungewöhnlich grossen Krystallen ungarischen Antimonglanzes wurden nun frische und vollkommen ebene Spaltungsplatten hergestellt, im kalten Zimmer (im Winter) behaucht, die Spitze einer heissen Nadel möglichst genau senkrecht aufgesetzt, bis die entsprechende Ellipse, innerhalb deren die Feuchtigkeit verdunstet ist, eine genügende Grösse und Schärfe angenommen hatte, dann Lycopodiumsamen aufgestreut und nach raschem Umkehren der auf das Innere der Ellipse gefallene Theil desselben abgeklopft. Der ganze Vorgang - das Anhauchen der Fläche, die Erzeugung der Figur, das Bestreuen und nachherige Abschutteln des Pulvers - beansprucht nur eine Zeitdauer von ungefähr fünf Secunden.

Die Figuren besassen eine ausgeprägt elliptische Form. Die längere Axe der Ellipse verlief parallel der $c$-Axe des Krystalles. In Fig. 18 ist der zu dem Versuche verwendete Krystall in ca. $\frac{2}{3}$ naturlicher Grösse mit einer der grösseren Ellipsen dargestellt. Um festzustellen, ob die längere Axe der Ellipse genau parallel der verticalen Axe des Krystalles verlaufe, wurde folgendes Verfahren eingeschlagen: Der Krystall mit einer der besten unter den erzeugten Figuren wurde auf dem horizontalen, drehbaren Objecttische des von P. Groth (s. dessen Physikal. Krystallographie 2. Aufl. S. 666) modificirten Elasticitätsapparates von Warburg und Koch befestigt. Einer der Fäden des Mikroskopes wurde auf die vollkommen geradlinige Krystallkante $c c^{\prime}$ eingestellt, und dann vermittelst der Mikrometerschrauben die Mitte des Fadenkreuzes mit dem Punkte zur Goincidenz gebracht, wo die Nadelspitze die Krystallfläche berührt hatte, worauf die Stellung des drehbaren Objecttisches abgelesen wurde. Vermittelst der dem verticalen Faden parallelen Mikrometerbewegung wurde dann der Schnittpunkt des Fadenkreuzes auf einen der Punkte verlegt, wo der Kreuzfaden $y$ den Rand der Ellipse schnitt und eine Ablesung der Mikrometerschraube vorgenommen, darauf zu dem gegenüberliegenden Punkte gerúckt, worauf abermals abgelesen wurde. Der Unterschied war gleich der Länge des grössten Durchmessers der Ellipse. In derselben Weise wurde die Länge des kleinsten Durchmessers bestimmt. Der Krystall wurde nun um $5^{0}$ nach links gedreht und der längere, als $y^{\prime}$ bezeichnete Durchmesser, der mit dem ersteren Durchmesser einen Winkel von $5^{0}$ bildete, gemessen. In derselben Weise wurde eine Reihe von funf Durchmessern, die je $5^{0}$ von einander abstanden, an jeder Seite von $y$ gemessen. Nachstehend folgen die sich auf drei Ellipsen beziehenden Messungen : 


$$
\begin{aligned}
& y=0,22 \mathrm{~mm} \\
& -y^{\prime}=6,22 \quad y^{\prime}=6,23 \\
& -y^{\prime \prime}=6,15 \quad y^{\prime \prime}=6,13 \\
& -y^{\prime \prime \prime}=6,05 \quad y^{\prime \prime \prime}=6,04 \\
& -y^{\mathrm{IV}}=5,89 \quad y^{\mathrm{IV}}=5,91 \\
& -y^{v}=5,70 \quad y^{\vee}=5,72 \\
& y=3,21 \mathrm{~mm} \\
& -y^{\prime}=3,17 \quad y^{\prime}=3,17 \\
& -y^{\prime \prime}=3,15 \quad y^{\prime \prime}=3,16 \\
& -y^{\prime \prime \prime}=3,13 \quad y^{\prime \prime \prime}=3,13 \\
& -y^{\mathrm{IV}}=3,11 \quad y^{\mathrm{IV}}=3,09 \\
& -y^{\mathbf{v}}=3,05 \quad y^{\mathbf{v}}=3,03 \\
& y=5,48 \mathrm{~mm} \\
& -y^{\prime}=5,47 \quad y^{\prime}=5,48 \\
& -y^{\prime \prime}=5,41 \quad y^{\prime \prime}=5,42 \\
& -y^{\prime \prime \prime}=5,33 \quad y^{\prime \prime \prime}=5,30 \\
& -y^{\mathrm{IV}}=5,16 \quad y^{\mathrm{IV}}=5,17 \\
& -y^{\mathrm{v}}=5,06 \quad y^{\mathrm{V}}=5,04
\end{aligned}
$$

Diese Messungen deuten ohne Zweifel darauf hin, dass $y$, die langere Axe der Ellipse, der Krystallkante $c c^{\prime}$ und daher auch der krystallographischen Axe $c$ parallel liegt, da die entsprechenden Durchmesser der Ellipse an jeder Seite von $y$ symmetrisch zu $y$ abnehmen. Hieraus ist der Schluss zu ziehen, dass die Wärmeleitungsfiguren des Antimonit auf ein durchaus rhombisches Mineral hinweisen.

Was das Verhältniss zwischen den beiden Axen der Ellipse betrifft, so ergaben die nachstehend angefuhrten zehn Messungsreihen eine Aenderung, die der Grösse der Ellipse entspricht, indem die grösseren Ellipsen die relativ grössere Verschiedenheit der Axen aufweisen. Wie in dem oben citirten Aufsatze von Röntgen dargethan wird, ist dies so zu erklären, dass die anfangs von "der Nadel durch die Luft ausströmende Wärme auf die Ellipse eine Einwirkung austubt, die sich darin äussert, dass die Ellipse eine gleichmässiger kreisförmige Gestalt annimmt.
(1) $2, \frac{y}{2,72}: \stackrel{x}{2,03}=1,339$
(2) $2,75: 2,01=1,368$
(3) $2,94: 2,27=1,295$.
(4) $3,15: 2,38=1,327$
(5) $3,21: 2,42=1,322$
(6) $3,33: 2,46=1,353$
(7) $3,84: 2,88=1,326$
(8) $4,22: 3,05=1,383$
(9) $5,48: 3,92=1,397$
(10) $6,22: 4,40=1,436$ 
Nebenbei wurde noch ein weiterer Beweis für die rhombische Natur des Antimonit durch die Druckfiguren geliefert, die von der zur Erzeugung der Ellipsen verwendeten Nadelspitze hervorgebracht wurden (Fig. 18 bei $b$ und auf beistehendem Holzschnitte). Man könnte dieselben als Rechtecke mit einspringenden Winkeln an jedem Ende bezeichnen. Die Figuren stehen ihrer Längsseite nach rechtwinkelig zur verticalen Axe und folglich auch zu der längeren Axe der Wärmeleitungsellipse (vgl.

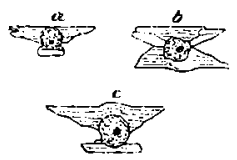
Fig. $18 b$ ). Wird die Nadel scbräg gehalten, so entsteht nur die Hälfte des bei einer normalen Haltung der Nadel zur Fläche erzeugten Bildes (vgl. beistehende Fig. $a$ und $c$ ).

\section{Wärmeleitung des Bournonit.}

Die Versuche, die mit Bournonit angestellt wurden, waren weniger befriedigend, als diejenigen mit Antimonit, erstens wegen der geringeren Grösse der Krystalle, die eine entsprechende Kleinheit der Ellipsen und daher minder genaue Resultate bedingte; zweitens wegen des gleichmässig unebenen Gharakters der Bournonitfläche $\{001\}$, die allein bei den zur Verfugung stehenden Krystallen zur Vornahme der Versuche hinreichend gross war.

Der Krystall, der sich am besten zu dem vorliegenden $Z$ wecke eignete, befand sich unter den Krystallen aus Přibram (Krystall Nr. 2, Fig. 1). Die Figuren, die in diesem Falle gewonnen wurden, waren ebenfalls Ellipsen, wichen aber nur um einen so geringen Betrag von der Kreisform ab, dass das unbewaffnete Auge sie kaum von letzterer zu unterscheiden vermochte. Durch die Messungen wurden sie aber in jedem einzelnen Falle als wirkliche Ellipsen ausgewiesen, deren längere Axe parallel der zwischen dem Brachydoma $n\{011\}$ und dem Brachypinakoid $b\{010\}$ liegenden Kante verlief. Fig. 19 zeigt in dreifacher Vergrösserung den eben erwähnten Krystall von Přibram, mit einer der besten Ellipsen, die auf der Fläche $\{001\}$ erzeugt werden konnten. Der durch die Unebenheit der Fläche bedingte unregelmasssige Ellipsenrand gestattete nicht so genaue Messungen, wie sie beim Antimonit angestellt wurden. Es wurde eine Reihe von 36 Durchmessern, die um Winkel von $5^{0}$ von einander abstanden und die Ellipse in 72 Segmente theilten, gemessen. Der Durchschnitt von $y$ und $y^{\prime}$ mit fünf zwischen, ihnen liegenden Durchmessern, die mit einander einen Winkel von $30^{\circ}$ bildeten, wurde als Werth von $y^{\prime}$ angenommen, in der gleichen Weise ein Durchschnitt der zwischen $y$ bis inclusive $-y^{\prime}$ liegenden Durchmesserwerthe als Werth von $y^{\prime}$, und ein Durchschnitt der zwischen $x$ und $x^{\prime}$, und $x$ und $-x^{\prime}$ liegenden als Werth von $x^{\prime}$ bezw. $-x^{\prime}$.

Die Resultate sind mit den gesonderten Werthen von $x$ und $y$ nachstehend mitgetheilt : 


$$
\begin{array}{rlrl}
y & =1,93 \mathrm{~mm} & x & =1,86 \mathrm{~mm} \\
y, y^{\prime} & =1,928 & x, x^{\prime} & =1,895 \\
y,-y^{\prime} & =1,928 & x,-x^{\prime} & =1,882
\end{array}
$$

Nach diesen Messungen sind $y^{\prime}$ und $-y^{\prime}$ gleich, während der Unterschied von $x^{\prime}$ und $-x^{\prime}$ nur $0,013 \mathrm{~mm}$ beträgt, woraus hervorgeht, dass um jede der beiden Ellipsenaxen ein Symmetrieverhältniss besteht.

Im Folgenden ist ein Vergleich der Verhältnisse $y: x$ und $x: y$ gegeben, wie sie aus den an funf verschiedenen Ellipsen vorgenommenen Messungen dieser Durchmesser sich ergaben :

$$
\begin{aligned}
& y \quad x \quad \text { Verhältniss: } \\
& \text { (1) } 1,63: 1,57=1,0382 \\
& \text { (2) } 1,64: 1,54=1,0648 \\
& \text { (3) } 1,79: 1,65=1,0848 \\
& \text { (4) } 1,92: 1,85=1,0387 \\
& \text { (5) } 1,93: 1,86=1,0376 \\
& x \quad y \quad \text { Verbältniss: } \\
& \text { (1) } 1,57: 1,63=0,9680 \\
& \text { (2) } 1,54: 1,64=0,9390 \\
& \text { (3) } 1,65: 1,79=0,9217 \\
& \text { (4) } 1,85: 1,92=0,9632 \\
& \text { (5) } 1,86: 1,93=0,9637
\end{aligned}
$$

Wegen der geringfugigen Unterschiede in der Grösse der Ellipsen sowohl, wie wegen der minderen Beschaffenheit der letzteren konnte nicht festgestellt werden, ob, wie im Falle von Antimonit, das Verhältniss der Axen mit der Grösse in annăhernd gleichem Maasse wechselt.

In der zweiten Verhältnissreihe $x: y$ ist eine ziemlich nahe Uebereinstimmung mit dem Verhältnisse der krystallographischen Axen $a$ und $b$ $(a: b=0,9379)$ zu constatiren, da aber $x$ parallel zur Axe $b$ und $y$ paralle] zur Axe $a$ ist, so liegt das Verbältniss der durch $x$ und $y$ dargestellten Werthe der Warrmefortpflanzung nahe dem umgekehrten Verhältnisse der krystallographischen Axen, zu denen sie parallel stehen. 